\title{
Paracoccidioidomicosis pulmonar. Reporte de dos casos
}

\section{Pulmonary paracoccidioidomycosis. Report of two cases}

\author{
Héctor Andrés Castañeda Camacho, MD. ${ }^{(1)}$; Diana Jimena Cano Rosales, MD.(2)
}

\section{Resumen}

La paracoccidioidomicosis o blastomicosis sudamericana, es una patología micótica sistémica, progresiva y crónica, generada por el agente Paracoccidioides brasiliensis, enfermedad exclusiva de América Latina y que representa un problema de salud pública relevante.

Se exponen dos casos clínicos, correspondientes a pacientes del género masculino, adultos promedio, agricultores, con hemoptisis y pérdida anormal de peso, radiográfias con patrón nodular y tomografia de tórax con imágenes en vidrio esmerilado y nódulos cavitados. En ambos casos se realizó fibrobroncoscopia, con lavado broncoalveolar, además de coloración histoquímica de Gomori que mostró levaduras micóticas esféricas grandes y pequeñas, con formaciones características de "timón de barco", morfológicamente compatibles con paracoccidioidomicosis.

Los factores epidemiológicos, correlacionados con una historia clínica completa y con los hallazgos radiológicos, junto con la fibrobroncoscopia y la histoquímica compatible, llevan al diagnóstico y a la confirmación de paracoccidioidomicosis pulmonar, lo que permite iniciar un manejo oportuno y adecuado con el objetivo de prevenir secuelas significativas.

Palabras clave: paracoccidioidomicosis pulmonar, hallazgos imagenológicos, presentación clínica, epidemiología.

\begin{abstract}
Paracoccidioidomycosis, or South American blastomycosis, is a systemic, progressive, and chronic fungal disease, caused by Paracoccidioides brasiliensis. This disease is to be found only in Latin America, and is a relevant public health concern.

We expose two clinical cases, both of male adult farmers who presented with hemoptysis and abnormal weight loss. Radiographs showed nodular patterns, and computed tomographies of the chest showed ground-glass opacities and cavitary nodules. In both cases, bronchoscopy with bronchoalveolar lavage was carried out, plus Gomori histochemical stain that showed large and small spherical mycotic yeasts, with characteristic "ship's wheel" formations, consistent with paracoccidioidomycosis.

Epidemiological factors, together with a complete clinical history and radiological findings, plus bronchoscopy with consistent histochemistry, lead to diagnosis and confirmation of pulmonary paracoccidioidomycosis, which allows for timely and adequate management, with the aim of preventing significant sequelae.
\end{abstract}

Keywords: pulmonary paracoccidiodomycosis, radiological findings, clinical presentation, epidemiology.
${ }^{(1)}$ Residente de segundo año de Medicina
Interna, Universidad Autónoma de Buca-
ramanga. Bucaramanga, Colombia.
${ }^{(2)}$ Especialista en Medicina Interna, Fellow
de Neumología, Universidad de la Sabana.
Bogotá, Colombia.
Correspondencia: Héctor Andrés Castañe-
da Camacho, correo electrónico: handresca@
hotmail.com
Recibido: $20 / 05 / 16$. Aceptado: 10/01/17. 


\section{Caso 1}

Paciente de género masculino, de 56 años, agricultor, con clínica de 6 meses de tos con expectoración sanguinolenta, asociada a perdida de $6 \mathrm{~kg}$ de peso, adinamia, aparición de masas en el cuello, fiebre subjetiva ocasional; 15 días antes de consultar presentó disnea de pequeños esfuerzos y secreción nasal fétida hialina. Como antecedente cirrosis hepática alcohólica (Child-Pugh B) y varices esofágicas grado II. En consultas previas por sintomatología respiratoria similar y ocupación alveolar en radiografía de tórax consideraron neumonía basal derecha, para lo cual recibió tratamiento sin mejoría de los síntomas.

Al examen físico se encontraron signos vitales normales PA 116/80 mm Hg, FC 72 latidos por minuto, FR 16 respiraciones por minuto, T $36,5{ }^{\circ} \mathrm{C}$, $\mathrm{SatO}_{2}$ al ambiente $94 \%$, ganglios de $2-3 \mathrm{~cm}$ diámetro fibroelásticos, no dolorosos en hemicuello derecho zona II, III y V, con ruidos respiratorios normales sin agregados, ascitis leve, edema grado II con fóvea, tinte ictérico en escleras y examen neurológico sin alteraciones.
Paraclínicos: creatinina: 0,73 mg/dl, BUN: 6,9 $\mathrm{mg} / \mathrm{dl}$, bilirrubina total $1,4 \mathrm{mg} / \mathrm{dl}$, directa $0,99 \mathrm{mg} /$ dl, AST $32 \mathrm{UI} / \mathrm{L}$, ALT $15 \mathrm{UI} / \mathrm{L}, \mathrm{Na}: 133 \mathrm{mmol} / \mathrm{l}$, $\mathrm{K}: 3,78 \mathrm{mmol} / \mathrm{l}, \mathrm{Cl}: 96,8 \mathrm{mmol} / \mathrm{l}, \mathrm{Hb}: 10,3 \mathrm{~g} / \mathrm{dl}$, VCM: 96 fl, RDW: 14,2\%. Leucocitos: 7.580/ $\mathrm{mm}^{3}$, Neutrófilos: $67 \%$, Linfocitos: 10,4\%, Eosinófilos 14,2\%, Plaquetas: $152.000 / \mathrm{mm}^{3}$, PT: 12,9 s, INR: 1,27, TPT: 38,3 s, PCR 35,85, albúmina 2,45 g/dl, Gases arteriales: $\mathrm{pH}: 7,46, \mathrm{PaCO}_{2}: 30,8$ $\mathrm{mm} \mathrm{Hg} \mathrm{PaO}: 76,3 \mathrm{~mm} \mathrm{Hg}, \mathrm{HCO}_{3}: 21 \mathrm{mmol} / 1, \mathrm{BE}$ : -1. mmol/1, VIH prueba presuntiva: negativa. Radiografía de tórax con múltiples lesiones nodulares en ambos campos pulmonares y opacidades reticulares en la base pulmonar derecha, aumento del hilio pulmonar derecho (figura 1).

La tomografía de tórax contrastada mostró imágenes nodulares cavitadas, vidrio esmerilado, densidad de partes blandas y bordes espiculados, de distribución difusa (figura 2).

En la fibrobroncoscopia se revisó árbol bronquial bilateral, así como las divisiones lobares y segmentarias; se encontraron mucosas infiltradas, sangrado y pigmentos de antracosis en los bronquios segmentarios. El Lavado broncoalveolar

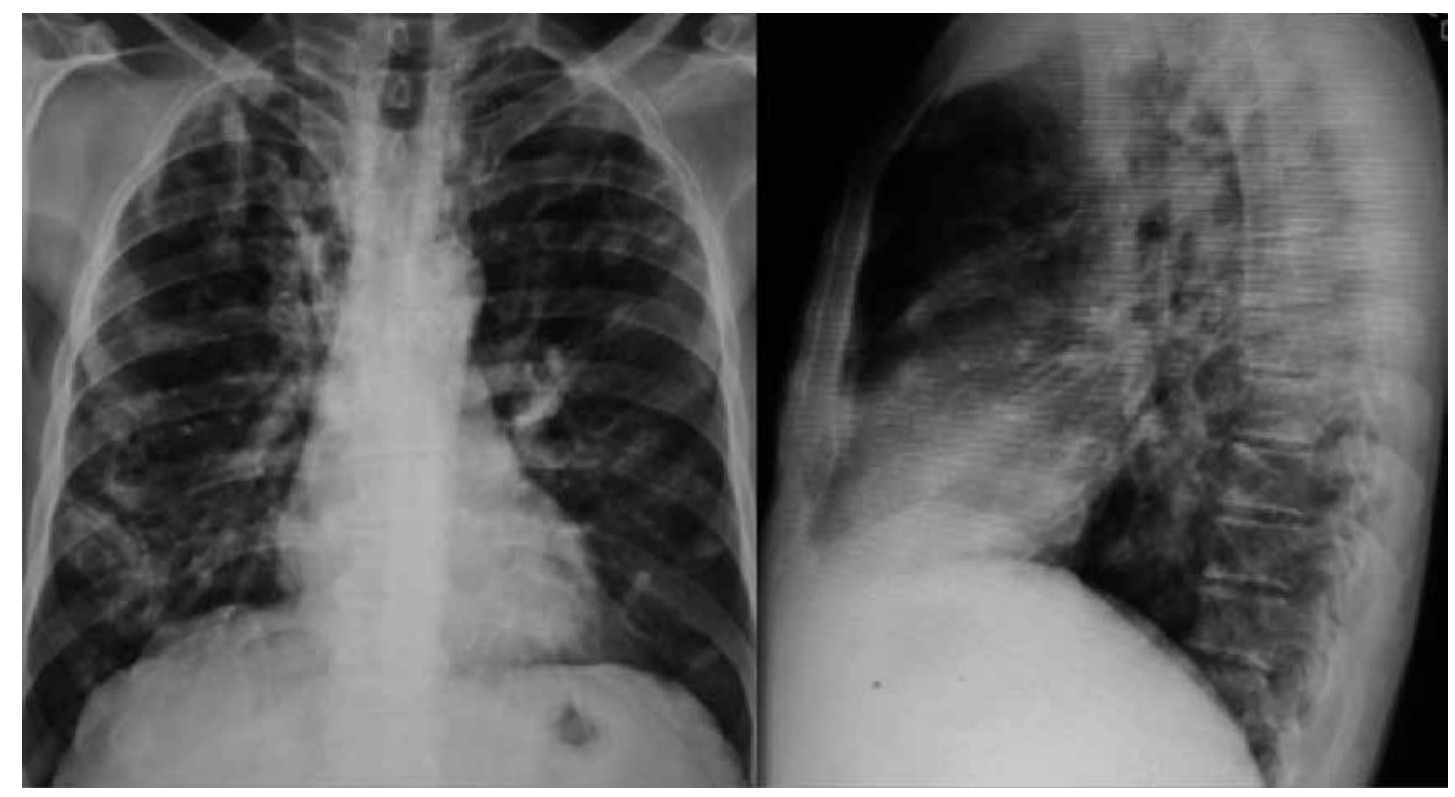

Figura 1. Múltiples lesiones nodulares en ambos campos pulmonares y opacidades reticulares en la base pulmonar derecha; aumento de hilio pulmonar derecho. 


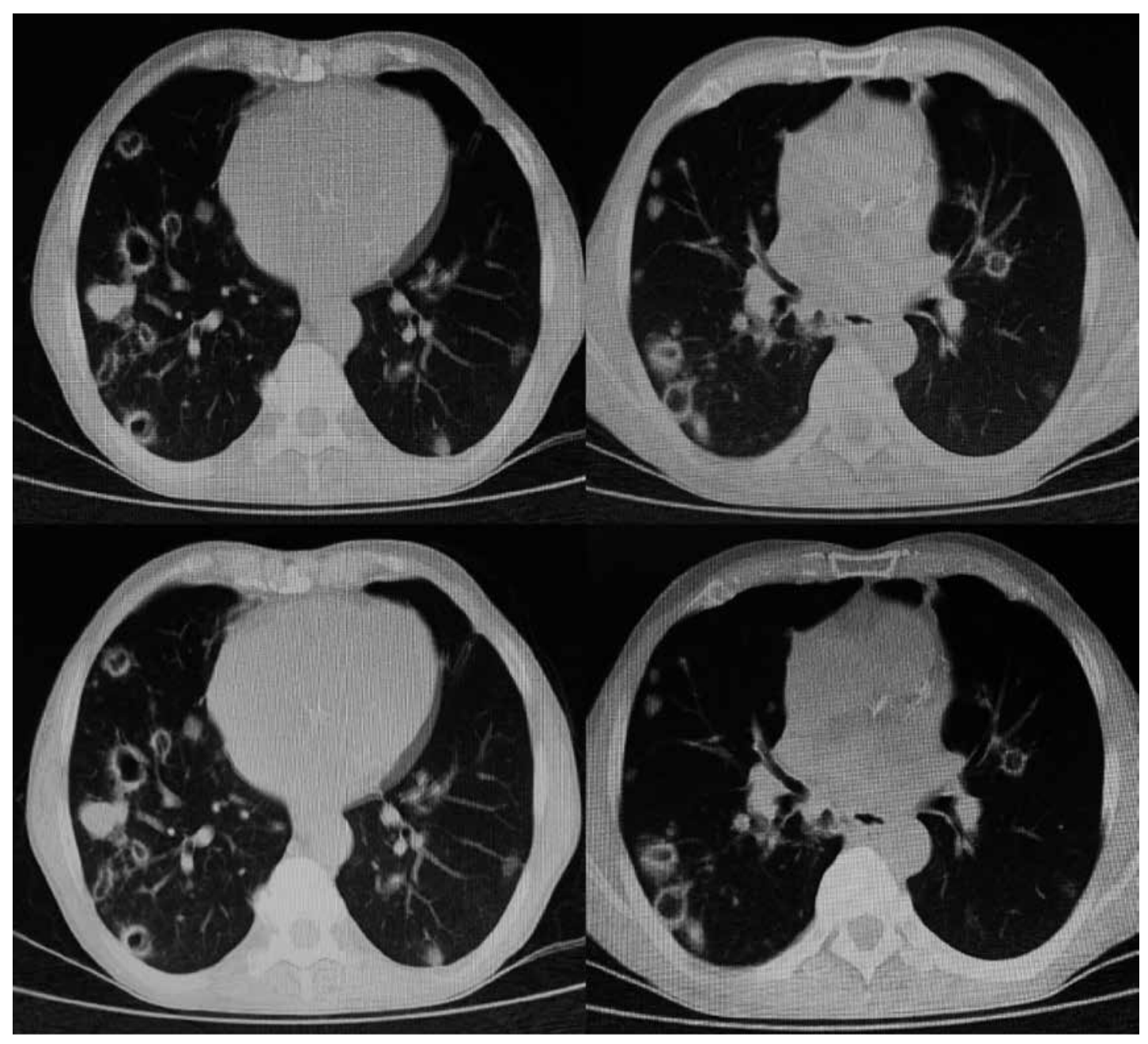

Figura 2. TAC de tórax: imágenes nodulares cavitadas con densidad de partes blandas, con bordes espiculados, de distribución difusa en ambos campos pulmonares, patrón en vidrio esmerilado, adenopatías mediastinales paraaórticas, paratraqueales y precarinales.

con la coloración histoquímica de Gomori resaltó frecuentes levaduras micóticas esféricas grandes y pequeñas, las grandes con yemas pequeñas con bases de gemación angosta y formaciones características en forma de "timón de barco" compatibles con paracoccidioidomicosis. La coloración de histoquímica de ZN no evidenció BAAR. Sin evidencia de malignidad.

Se diagnosticó paracoccidioidomicosis pulmonar y se inició itraconazol $200 \mathrm{mg}$ /día en suspensión por mejor biodisponibilidad oral, para manejo por un año; se vigiló la función hepática de manera frecuente dado el antecedente de cirrosis alcohólica.

\section{Caso 2}

Paciente de 55 años, de género masculino, agricultor, sin antecedentes crónicos de relevancia, previamente sano, antecedente de contacto con paciente diagnosticado con tuberculosis pulmonar, con cuadro clínico de tres meses de tos con expectoración hemoptoica, pérdida de $10 \mathrm{~kg}$ de peso, diaforesis nocturna, disnea de medianos esfuerzos, fiebre subjetiva intermitente, manejado con antibioticoterapia por sospecha de neumonía; aportó cultivo de esputo positivo para Klebsiella pneumoniae multisensible y baciloscopias negativas. La figura 3 corresponde a la radiografía de tórax al momento 


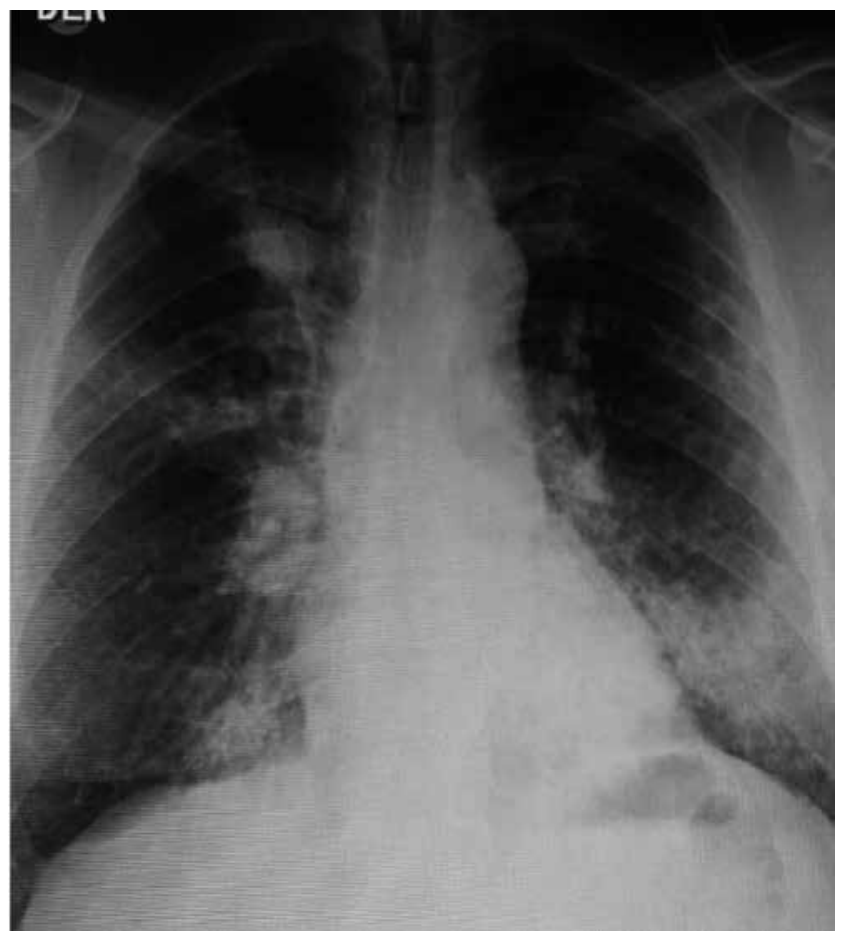

Figura 3. Infiltrados mixtos perihiliares y en ambas bases, con tendencia a la consolidación; imágenes pseudonodulares en base y ápice pulmonar derecho.

de nuestra valoración; se observan infiltrados mixtos perihiliares y en ambas bases, con tendencia a la consolidación e imágenes pseudonodulares en base y ápice pulmonar derecho.

$\mathrm{Al}$ examen físico signos vitales normales PA 110/70 mm Hg, FC 82 latidos por minuto, FR 15 respiraciones por minuto, $\mathrm{T} 37,2^{\circ} \mathrm{C}$, $\mathrm{SatO}_{2}$ al ambiente $95 \%$, sin adenopatías cervicales, ruidos respiratorios con estertores en base pulmonar izquierda.

Paraclínicos: creatinina $0,79 \mathrm{mg} / \mathrm{dl}$ BUN 14,8 $\mathrm{md} / \mathrm{dl}, \mathrm{Hb} 14,3 \mathrm{~g} / \mathrm{dl}$ Leucocitos: $12.430 / \mathrm{mm}^{3}$, Neutrófilos: $71 \%$, Linfocitos: 18\%, Eosinófilos 1\%, Plaquetas 415.000/. $\mathrm{mm}^{3}$, PCR 83,48, Procalcitonina 0,08 , VIH prueba presuntiva: negativa.

La tomografía de tórax contrastada mostró múltiples lesiones en parches en vidrio esmerilado con broncograma aéreo y lesiones cavitadas especialmente en los lóbulos superiores. En el mediastino se observaron adenopatías pequeñas en la ventana aórtico-pulmonar de menos de $1 \mathrm{~cm}$, además de algunas en la región precarinal e infracarinal (figura 4).

Se realizó fibrobroncoscopia en la que se observó árbol bronquial bilateral con mucosa de aspecto y calibre normal, con toma de muestra donde la coloración histoquímica de Gomori resaltó levaduras esféricas grandes y pequeñas, las grandes con yemas pequeñas con bases de gemación angosta con formaciones características en forma de "timón de barco", morfológicamente compatibles con paracoccidioidomicosis (figura 5). Coloración $\mathrm{Zn}$ no evidencia BAAR. Negativo para malignidad.

Se inició manejo con itraconazol oral durante 12 meses.

\section{Discusión}

La paracoccidioidomicosis es una micosis endémica causada por el hongo difórmico Paracoccidioides brasiliensis, exclusiva de América Latina, con mayor prevalencia en América del Sur, donde Colombia ocupa el segundo lugar. Afecta más a hombres de 30 a 50 años, dedicados a actividades agrícolas $(1,2)$.

El hongo tiene la capacidad de multiplicarse en los tejidos profundos del huésped, donde puede crecer al ambiente en algunos tipos de entornos propicios (3). Este se adquiere por inhalación de las formas infectantes causando micosis sistémica. La enfermedad tiene largos periodos de latencia, lo cual ha sido demostrado por casos fuera de zonas endémicas, y sugiere una reactivación endógena. Se subdivide en dos grupos que se diferencian por la evolución y edad del huésped: la forma juvenil, que tiene un cuadro clínico agudo o subagudo con distribución predominantemente en el sistema reticuloendotelial y linfático, y la forma crónica o de reactivación, que tiene un curso más insidioso en el que el pulmón es el órgano más afectado (4).

En los casos expuestos, los pacientes compartían características epidemiológicas, género, edad promedio, ocupación, cuadro de sintomatología respiratoria, asociado a pérdida de peso, fiebre y hemoptisis; uno de ellos con adenopatías, 


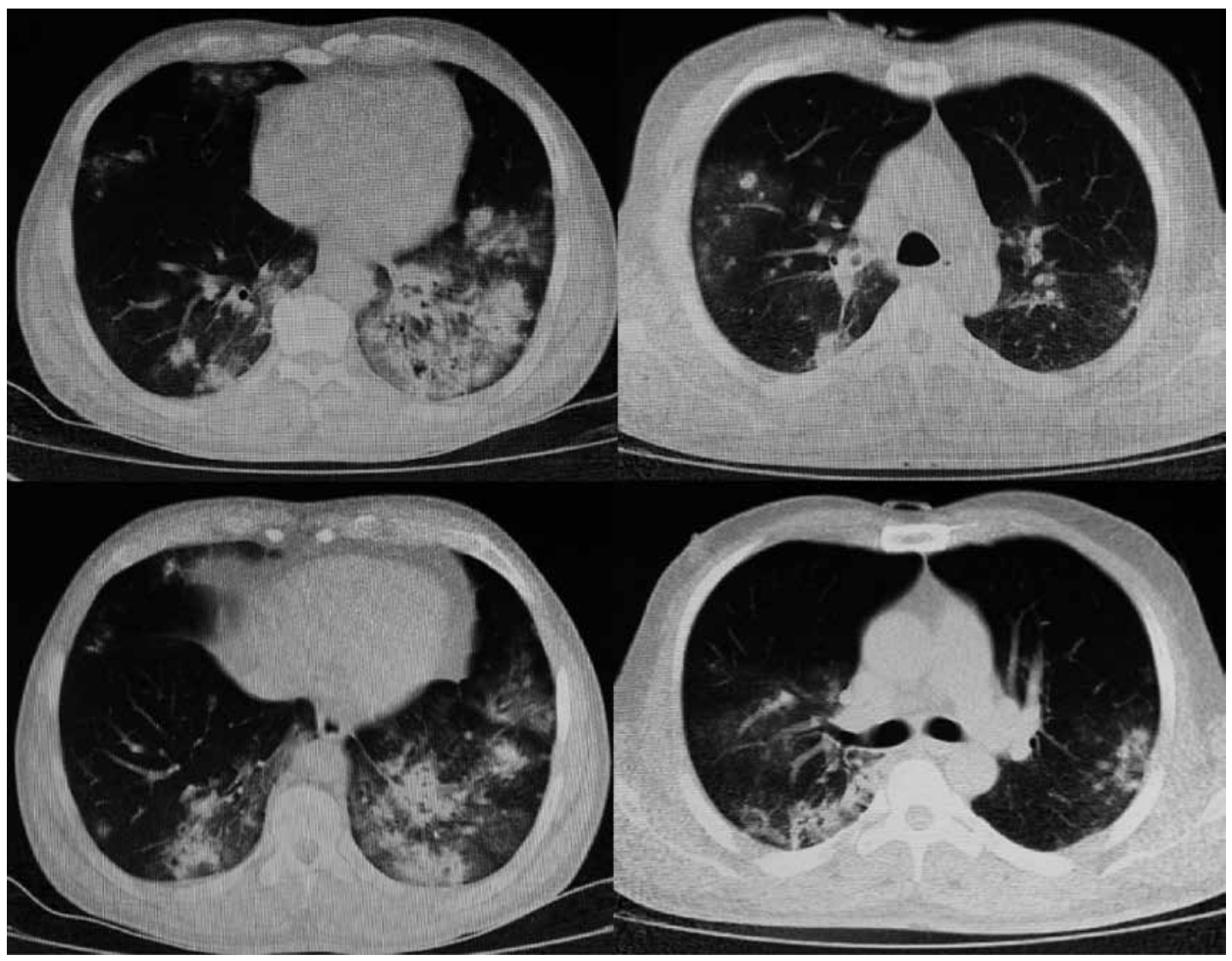

Figura 4. TAC de tórax contrastado con múltiples lesiones en parches en vidrio esmerilado, con broncograma aéreo y lesiones nodulares cavitadas especialmente en los lóbulos superiores.

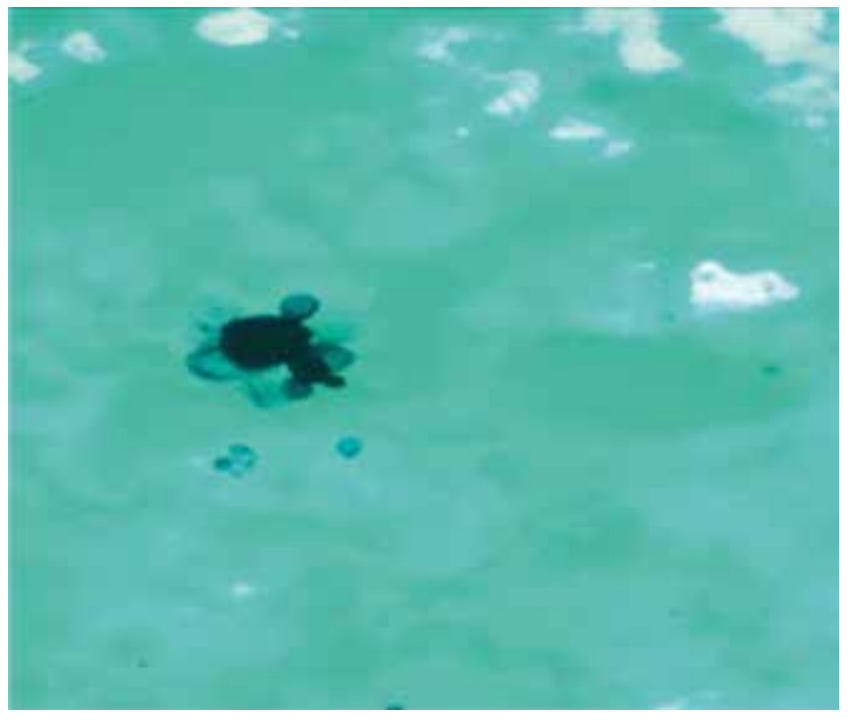

Figura 5. Coloración Gomori de uno de los casos, con imagen en timón de barco, compatible con paracoccidioidomicosis pulmonar. inicialmente tratadas bajo diagnóstico de neumonía sin mejoría con antibioticoterapia. La clínica manifestada en esto es compatible con la forma crónica progresiva de la micosis, forma que se encuentra hasta en un $90 \%$ de los casos (5). Se destacan además los pocos hallazgos vistos a la auscultación pulmonar, que no se correlacionan con las variadas lesiones imagenológicas encontradas.

En cuanto a la radiografía de tórax en pacientes con paracoccidioidomicosis, un $64 \%$ cursa con infiltrados intersticiales, como se describió en el segundo caso, además de lesiones mixtas nodulares y alveolares que tienden a ser simétricas, bilaterales con predominio en los campos centrales e inferiores (6), características que se encontraron en ambos pacientes, aunque también con una distribución variable sin obedecer a un patrón definido 
tal y como lo reporta la literatura (7). Estas anomalías radiográficas son muy cardinales en el diagnóstico de enfermedades como tuberculosis y paracoccidioidomicosis; esta última siempre debe incluirse en el diagnóstico diferencial de pacientes con antecedentes exposicionales y que habitan en áreas endémicas (8).

Igualmente, en los dos casos se hizo TAC de tórax que mostró imágenes en vidrio esmerilado, que es el hallazgo tomográfico más común, especialmente en quienes no han recibido tratamiento (7), seguido por pequeños nódulos centrolobulillares, nódulos cavitados, áreas de enfisema cicatricial, engrosamiento septal lobular y distorsión de la arquitectura. La combinación de todos estos hallazgos puede observarse en la mayoría de los casos reportados, y su presentación puede ser focal, multifocal o difusa (7-9), como ocurrió en estos pacientes.

La alta sospecha clínica e imagenológica de infección micótica, con un perfil epidemiológico compatible, lleva a la fibrobroncoscopia con lavado broncoalveolar, que confirma la presencia de levaduras con formaciones características en "timón de barco", que son morfológicamente compatibles con paracoccidioidomicosis. Los principales diagnósticos diferenciales son tuberculosis e histoplasmosis por su similitud clínica y radiológica, ambos descartados en los pacientes expuestos. El diagnóstico definitivo se da por aislamiento del hongo en biopsia, secreciones o cultivo de esputo; en casos los casos el hongo se evidenció por coloración de Gomori (10).

Se resalta, así mismo, la importancia del diagnóstico e inicio oportuno del manejo adecuado, ya que el daño pulmonar progresa a fibrosis y de ahí a hipoxemia, disfunción ventilatoria e insuficiencia suprarrenal. El pilar del tratamiento es el itraconazol, que es la mejor opción en la forma leve y moderada por 6-12 meses; el trimetoprim-sulfametoxazol es una alternativa, en tanto que la anfotericina B se reserva para las formas severas (11). En los casos descritos se inició manejo con itraconazol.

En Colombia la infección se ha reportado desde 1950 (12), convirtiéndose en una de la micosis sistémicas más frecuentes, seguida de la histoplasmosis (13). Teniendo en cuenta que el país cuenta con un área rural del $94 \%$, que de la población total un $32 \%$ habita dicha zona y que 9 de 32 departamentos se consideran áreas endémicas (Santander en segundo lugar luego de Antioquia) (14), adquiere relevancia el estudio y reporte de casos con esta patología para entender más su comportamiento y verdadera incidencia, con miras a establecer medidas de prevención y tratamiento dadas las secuelas que puede generar. Resulta interesante el factor protector de la infección en las mujeres, al parecer por la presencia de receptores 17-b- estradiol en el citoplasma de P. Brasiliensis; esta hormona femenina inhibe in vitro la transformación micelial a levadura del hongo (15), lo que puede explicar la mayor presencia de la enfermedad en hombres y a futuro abrir más campos de investigación y tratamiento. En la actualidad ya se han hecho estudios para desarrollar una vacuna humana contra la paracoccidioidomicosis (16).

\section{Bibliografía}

1. Torrado E, Castañeda E, De la Hoz F, Restrepo A. Paracoccidioidomicosis: Definición de las áreas endémicas de Colombia. Biomédica. 2000;20(2):327-34.

2. Wanke B, Abidon M. Paracoccidioidomycosis. J Bras Pneumol. 2009;35:1245-49.

3. Bagagli E, Theodoro RC, Bosco SM, McEwen JG. Paracoccidioides brasiliensis: phylogenetic and ecological aspects. Mycopathologia. 2008;165:197-207.

4. Costa AN, Benard G, Pereira AL, Fujita CL, Kono AS, Salge $\mathrm{JM}$, et al. The lung in paracoccidioidomycosis: new insights into old problems. Clinical Science. 2013;68(4):441-48.

5. Bocca AL, Amaral AC, Teixeira MM, Sato PK, ShikanaiYasuda MA, Soares Felipe MS. Paracoccidioidomycosis: eco-epidemiology, taxonomy and clinical and therapeutic issues. Future Microbiol. 2013;8(9):1177-91.

6. Bennett J, Dolin R, Blaser M. Principles and practice of infectious diseases. 8th. Edition. 2014:2996-8.

7. Barreto MM, Marchiori E, Amorim VB, Zanetti G, Takayassu TC, Escuissato DL, et al. Thoracic paracoccidioidomycosis: Radiographic and CT Findings. RadioGraphics. 2012;32:71-84.

8. Costa de Freitas RM, Prado R, Silva do Prado FL, Braga de Paula I, Alves Figueiredo MT, Ferreira CS, et al. Pulmonary paracoccidiodomycosis: radiology and clinical-epidemiological evaluation. Revista do Sociedade Brasileira de Medicina Tropical. 2010;43(6):651-56. 
9. Marchiori E, Valiante PM, Mauro Mano P, Zanetti G, Escuissato DL, Souza AS, et al. Paracoccidioidomycosis: High-resolution computed tomography - pathologic correlation. Eur J Radiol. 2011;77:80-4.

10. Arciniegas Quiroga W, Orjuela Zuluaga DL. Paracoccidioidomicosis crónica. Reporte de un caso. Investigaciones Andina. 2011;22(13):228-35.

11. Shikanai Yasuda MA. Paracoccidioidomycosis treatment. Rev Inst Med Trop. Sao Paulo. 2015;57(19):31-7.

12. Mendez-Lemaitre A. Blastomicosis suramericana y otras micosis en Colombia. Rev Hosp Samaritana. 1950;1:3-20.
13. Moriones Robayo CA, Guerra Ortiz CP. Histoplasmosis laríngea: reporte de primer caso en Colombia. Colombia Médica. 2014;45(4).

14. Torrado E, Castañeda E, de la Hoz H, Restrepo A. Paracoccidioidomicosis: definición de las áreas endémicas de Colombia. Biomédica. 2000:20:327-34.

15. Restrepo A, Salazar ME, Cano LE, Stover P, Feldaman D, Stevensa DA. Estrogens inhibit mycelium to yeast transformation in the fungus Paracoccidioides brasiliensis. Infect Immunol.1984;46:346-53.

16. Travassos LR, Taborda CP. Paracoccidioidomycosis vaccine. Human Vaccines \& Immunotherapeutics. 2012;8. 